\title{
Solutions of Fractional Differential Type Equations by Fixed Point Techniques for Multivalued Contractions
}

\author{
Hasanen A. Hammad $\mathbb{D}^{1},{ }^{1}$ Hassen Aydi $\mathbb{D}^{2,3,4}$ and Manuel De la Sen ${ }^{5}{ }^{5}$ \\ ${ }^{1}$ Department of Mathematics, Faculty of Science, Sohag University, Sohag 82524, Egypt \\ ${ }^{2}$ Institut Supérieur d'Informatique et des Techniques de Communication, Université de Sousse, H. Sousse, Tunisia \\ ${ }^{3}$ Department of Mathematics and Applied Mathematics, Sefako Makgatho Health Sciences University, Ga-Rankuwa, \\ South Africa \\ ${ }^{4}$ China Medical University Hospital, China Medical University, Taichung 40402, Taiwan \\ ${ }^{5}$ Institute of Research and Development of Processes, University of the Basque Country, 48940- Leioa (Bizkaia), Spain
}

Correspondence should be addressed to Hassen Aydi; hassen.aydi@isima.rnu.tn

Received 16 June 2020; Revised 19 January 2021; Accepted 8 February 2021; Published 23 February 2021

Academic Editor: Atila Bueno

Copyright (C) 2021 Hasanen A. Hammad et al. This is an open access article distributed under the Creative Commons Attribution License, which permits unrestricted use, distribution, and reproduction in any medium, provided the original work is properly cited.

\begin{abstract}
This paper involves extended $b$-metric versions of a fractional differential equation, a system of fractional differential equations and two-dimensional (2D) linear Fredholm integral equations. By various given hypotheses, exciting results are established in the setting of an extended $b$-metric space. Thereafter, by making consequent use of the fixed point technique, short and simple proofs are obtained for solutions of a fractional differential equation, a system of fractional differential equations and a two-dimensional linear Fredholm integral equation.
\end{abstract}

\section{Introduction and Preliminaries}

In the last years, the fractional calculus branch $[1,2]$ has attracted great interest. There exist many kinds of proposed fractional operators, for instance, we have the well-known Caputo, Riemann-Liouville, Grunwald-Letnikov derivative etc. Among all the papers dealing with fractional derivatives, fractional differential equations as an important research field have attained great deal of attention from many researchers (see [3-8]).

There are many applications of the fractional topic in complex analysis, such as, in the sense of conformable derivatives and integrals, interesting results for fractional formulations of complex-valued functions of a real variable have been successfully introduced, which in turn open the door to the researchers to construct the theory of conformable integration by studying functions of a complex variable [9]. On the other hand, the standard definition for the Atangana-Baleanu fractional derivative involves an integral transform with a Mittag-Leffler function, where the kernel can be rewritten as a complex contour integral, which can be used to provide an analytic continuation of the definition to complex orders of differentiation [10]. These lines are very important due to their applications in the field of natural science or engineering.

In the last few decades and in the branch of fractional differential equations, Riemann-Liouville and Caputo derivative ones are the mostly used. Note that several fractional differential equations have been resolved by using fixed point techniques. This paper is concerned with this fact when considering the class of extended $b$-metric spaces.

Let $(\Re, \bowtie)$ be a metric space. Denote by $C B(\Re)$ a set of nonempty closed bounded subsets of $\Re$. Define the function $¥: C B(\Re) \times C B(\Re) \longrightarrow \mathbb{R}^{+}$by

$$
¥\left(\mho_{1}, \mho_{2}\right)=\max \left\{\sup _{\xi \in \sigma_{1}} \oplus\left(\xi, \mho_{2}\right), \sup _{\zeta \in \sigma_{2}} \oplus\left(\zeta, \mho_{1}\right)\right\},
$$

where $\oplus\left(\xi, \widetilde{\mho}_{2}\right)=\inf \left\{\oplus(\xi, \zeta): \zeta \in \widetilde{\mho}_{2}\right\}$, for $\widetilde{\mho}_{1}, \widetilde{\mho}_{2} \in C B(\Re)$. 
Then, $¥$ is called the Hausdorff-Pompeiu metric. Consider

$$
\begin{aligned}
& \delta\left(\mho_{1}, \mho_{2}\right)=\sup \left\{\omega(\xi, \zeta): \xi \in \mho_{1}, \zeta \in \mho_{2}\right\}, \\
& D\left(\mho_{1}, \mho_{2}\right)=\inf \left\{\omega(\xi, \zeta): \xi \in \mho_{1}, \zeta \in \mho_{2}\right\} .
\end{aligned}
$$

The following can be deduced from the definition of $\delta$. For all $\widetilde{\sigma}_{1}, \mho_{2}, \widetilde{\sigma}_{3} \in C B(\Re)$, we have the following:
(a) $\delta\left(\mho_{1}, \mho_{2}\right)=\delta\left(\mho_{2}, \mho_{1}\right)$
(b) $\delta\left(\mho_{1}, \mho_{2}\right)=0$ iff $\widetilde{\delta}_{1}=\widetilde{\mho}_{2}=\{\xi\}$
(c) $\delta\left(\mho_{1}, \mho_{3}\right) \leq \delta\left(\mho_{1}, \mho_{2}\right)+\delta\left(\mho_{2}, \mho_{3}\right)$
(d) $\delta\left(\mho_{1}, \mho_{1}\right)=\operatorname{diam} \mho_{1}$

In 2017, the concept of extended $b$-metric spaces has been initiated by Kamran et al. [11], by considering a control function at the right-hand side of the triangular inequality.

Definition 1 (see [11]). Let $\mathfrak{R}$ be a nonempty set and $\theta: \mathfrak{R} \times$ $\Re \longrightarrow[1, \infty)$ be a given function. An extended $b$-metric is a function $\omega_{\theta}: \mathfrak{R} \times \mathfrak{R} \longrightarrow[0, \infty)$ such that, for all $\eta, \xi, \sigma \in \mathfrak{R}$, we have the following:

(1) $\Phi_{\theta}(\eta, \xi)=0 \Longleftrightarrow \eta=\xi$

(2) $\omega_{\theta}(\eta, \xi)=\omega_{\theta}(\xi, \eta)$

(3) $\varpi_{\theta}(\eta, \xi) \leq \theta(\eta, \xi)\left[\varpi_{\theta}(\eta, \sigma)+\varpi_{\theta}(\sigma, \xi)\right]$

This (generalized) metric space has attracted many researchers where many real applications have been resolved. For more details, see [12-15]. Some of the related topological concepts are as follows.

Definition 2 Let $\left(\Re, \varpi_{\theta}\right)$ be an extended $b$-metric space. Let $\left\{\xi_{m}\right\}_{m \geq 0}$ be a sequence in $\mathfrak{R}$.

(1) $\left\{\xi_{m}\right\}$ converges to some $\xi$ in $\mathfrak{R}$, if for each $\varepsilon>0$, there is $M=M(\varepsilon) \in \mathbb{N}$ so that $\Phi_{\theta}\left(\xi_{m}, \xi\right)<\varepsilon$ for each $m \geq M$

(2) $\left\{\xi_{m}\right\}$ is Cauchy, if for each $\varepsilon>0$, there is $M=M(\varepsilon) \in \mathbb{N}$ so that $\Phi_{\theta}\left(\xi_{m}, \xi_{n}\right)<\varepsilon$ for all $m, n \geq M$

(3) $\left(\mathfrak{R}, \oplus_{\theta}\right)$ is called complete if each Cauchy sequence is convergent

Lemma 1 (see [12]). Let $\left(\mathfrak{R}, \aleph_{\theta}\right)$ be an extended $b-$ metric space. If the sequence $\left\{\xi_{n}\right\}$ in $\Re$ is such that $\lim _{n, m \rightarrow \infty} \Phi_{\theta}\left(\xi_{n}, \xi_{m}\right) \leq(1 / \nabla)$, where $\nabla \in[0,1)$ and $0 \leq \varpi_{\theta}\left(\xi_{n}, \xi_{n+1}\right) \leq \nabla \varpi_{\theta}\left(\xi_{n-1}, \xi_{n}\right)$, then $\left\{\xi_{n}\right\}$ is Cauchy.

Example 1 (see [16]). Take $\mathfrak{R}=[0,1]$. Given $\theta: \mathfrak{R} \times \mathfrak{R} \longrightarrow[1, \infty)$ as $\theta(\nu, \mu)=((\nu \mu+1) /(\nu+\mu))$. Consider the extended b-metric $\varpi_{\theta}: \Re \times \mathfrak{R} \longrightarrow[0, \infty)$ so that

$$
\varpi_{\theta}(\nu, \mu)= \begin{cases}\frac{1}{\nu \mu}, & \text { for } \nu, \mu \in(0,1], \nu \neq \mu, \\ 0, & \text { for } \nu=\mu,\end{cases}
$$

and $\omega_{\theta}(0, \mu)=\omega_{\theta}(\mu, 0)=(1 / \mu)$ for $\mu \in(0,1]$.
Definition 3 (see [17]). Denote by $\Xi$ the set of functions $\Upsilon: \mathbb{R}^{+} \longrightarrow(0,1]$ such that

(i) $\mathbb{R}^{+}=\{\eta \in \mathbb{R}: \eta>0\}$

(ii) For any sequence $\left\{\eta_{n}\right\}_{n=0}^{\infty}, \Upsilon\left(\eta_{n}\right) \longrightarrow 1$ implies that $\eta_{n} \longrightarrow 0$ as $n \longrightarrow \infty$

Example 2. Given $\Upsilon: \mathbb{R}^{+} \longrightarrow(0,1]$ as

$$
\Upsilon(\hbar)= \begin{cases}1-\frac{\hbar^{3}}{2}, & \text { if } \hbar \leq 1, \\ \tau<1, & \text { if } \hbar>1 .\end{cases}
$$

Clearly, $\Upsilon \in \Xi$.

The manuscript is organized as follows. In Section 2, some fixed point results in the class of extended $b$-metric spaces have been provided. We also present some useful examples. By using fixed point techniques, we solve in Section 3 a fractional nonlinear differential equation, we ensure in Section 4 the existence of a unique solution of a system of nonlinear fractional differential equations, and in Section 5, we establish that a two-dimensional linear Fredholm integral equation has a unique solution. At the end, in Section 6, we give a conclusion.

\section{Main Theorems}

In this section, $\mathfrak{R}$ refers to an extended $b$-metric space equipped with the distance $\omega_{\theta}$. We begin with the following lemmas.

Lemma 2. Let $\left(\Re, \varpi_{\theta}\right)$ be an extended $b$-metric space with the function $\theta: \Re \times \Re \longrightarrow[1, \infty)$. For any $\mho_{1}, \mho_{2}, \mho_{3} \in C B(\Re)$ and $\xi, \zeta \in \Re$, the following assertions are valid:

(i) $\varpi_{\theta}\left(\xi, \sigma_{2}\right) \leq \varpi_{\theta}(\xi, \zeta)$ for $\zeta \in \mho_{2}$

(ii) $\delta\left(\widetilde{\mho}_{1}, \widetilde{\mho}_{2}\right) \leq ¥\left(\widetilde{\mho}_{1}, \widetilde{\mho}_{2}\right)$

(iii) $\varpi_{\theta}\left(\xi, \mho_{2}\right) \leq ¥\left(\mho_{1}, \mho_{2}\right)$

(iv) $¥\left(\widetilde{\delta}_{1}, \widetilde{\delta}_{1}\right)=0$

(v) $¥\left(\widetilde{\delta}_{1}, \widetilde{\delta}_{2}\right)=¥\left(\widetilde{\delta}_{2}, \widetilde{\delta}_{1}\right)$

(vi) $¥\left(\widetilde{J}_{1}, \widetilde{J}_{3}\right) \leq \theta\left(\widetilde{J}_{1}, \widetilde{J}_{3}\right)\left[¥\left(\widetilde{J}_{1}, \widetilde{J}_{2}\right)+¥\left(\widetilde{J}_{2}, \widetilde{J}_{3}\right)\right]$

(vii) $\varpi_{\theta}\left(\xi, \mho_{1}\right) \leq \theta\left(\xi, \mho_{1}\right)\left[\varpi_{\theta}(\xi, \zeta)+\varpi_{\theta}\left(\zeta, \mho_{1}\right)\right]$

Proof. The assertions (i)-(v) follow immediately by Czerwik [18] in $b$-metric spaces and (vi)-(vii) follow immediately by the definition of an extended $b$ - metric space and (1) with (2).

Lemma 3. Let $\left(\Re, \oplus_{\theta}\right)$ be an extended $b-$ metric space. Then, for all $\sigma_{1}, \mho_{2} \in C B(\Re), \mu \in \mho_{1}$ and $\hbar \geq 1$, there exists $\eta(\mu) \in \delta_{2}$ such that $\Phi_{\theta}(\mu, \eta) \leq \hbar ¥\left(\delta_{1}, \delta_{2}\right)$.

Proof. By a similar way as in the proof of Lemma 4 in [19], we get the result.

Now, we state and prove our main theorems. 
Theorem 1. Let $\left(\Re, \oplus_{\theta}\right)$ be a complete extended b-metric space and $\wp, \mathfrak{I}: \mathfrak{R} \longrightarrow C B(\mathfrak{R})$ be multivalued mappings satisfying, for all $\xi, \ell \in \mathfrak{R}$,

where

$$
\begin{aligned}
& \Delta(\xi, \ell)=\max \left\{\varpi_{\theta}(\xi, \ell), \frac{\varpi_{\theta}(\xi, \wp \xi)\left[1+\varpi_{\theta}(\ell, \Im \ell)\right]}{1+\varpi_{\theta}(\wp \xi, \Im \ell)}, \frac{\varpi_{\theta}(\ell, \wp \xi)\left[1+\varpi_{\theta}(\xi, \wp \xi)\right]}{1+\varpi_{\theta}(\xi, \ell)}\right\}, \\
& \Pi(\xi, \ell)=\min \left\{\varpi_{\theta}(\xi, \ell) \varpi_{\theta}(\xi, \wp \xi), \varpi_{\theta}(\xi, \wp \xi) \varpi_{\theta}(\ell, \Im \ell), \varpi_{\theta}(\ell, \wp \xi) \varpi_{\theta}(\xi, \Im \ell)\right\} .
\end{aligned}
$$

(1) $\Omega: \mathbb{R}^{+} \longrightarrow \mathbb{R}^{+}$is continuous and nondecreasing function so that $\Omega(\tau)=0$ iff $\tau=0$

(2) $\Lambda: \mathbb{R}^{+} \longrightarrow \mathbb{R}^{+}$is a continuous function so that $\Lambda(\tau)=0$ iff $\tau=0$

If $\lim _{n, m \rightarrow \infty} \Phi_{\theta}\left(\xi_{n}, \xi_{m}\right) \leq(1 / k)$ for $k>1$, then $\wp$ and $\mathfrak{\Im}$ have a unique common fixed point (cfp).
Proof. Let $\xi \in \Re$ be a fixed element. Define $\xi^{\circ}=\xi$, and let $\xi_{1} \in \wp_{\xi^{\circ}}$, by Lemma 3 , there exists $\xi_{2} \in \Im \xi_{1}$ such that $\varpi_{\theta}\left(\xi_{1}, \xi_{2}\right) \leq \kappa ¥\left(\wp_{\xi^{\circ}}, \Im \xi_{1}\right)$. For $\xi_{2} \in \Im \xi_{1}$, there is $\xi_{3} \in \wp \xi_{1}$ such that $\varpi_{\theta}\left(\xi_{2}, \xi_{3}\right) \leq \kappa ¥\left(\wp \xi_{1}, \Im \xi_{2}\right)$.

Continuing with the same manner, we have $\xi_{2 n+1} \in \wp \xi_{2 n}$, $\xi_{2 n+2} \in \mathfrak{\Im} \xi_{2 n+1}$. If $\xi_{2 n+1}=\xi_{2 n+2}$, then the sequence $\left\{\xi_{n}\right\}$ is Cauchy. Suppose that $\xi_{2 n+1} \neq \xi_{2 n+2}$. Then, by (3), we have

$$
\Omega\left(\kappa \varpi_{\theta}\left(\xi_{2 n+1}, \xi_{2 n+2}\right)\right) \leq \Omega\left(\kappa ¥\left(\wp \xi_{2 n}, \Im \xi_{2 n+1}\right)\right) \leq \Omega\left(\Delta\left(\xi_{2 n}, \xi_{2 n+1}\right)\right)-\Lambda\left(\Pi\left(\xi_{2 n}, \xi_{2 n+1}\right)\right),
$$

where

$$
\begin{aligned}
& \Delta\left(\xi_{2 n}, \xi_{2 n+1}\right)=\max \left\{\begin{array}{c}
\varpi_{\theta}\left(\xi_{2 n}, \xi_{2 n+1}\right), \frac{\varpi_{\theta}\left(\xi_{2 n}, \wp \xi_{2 n}\right)\left[1+\varpi_{\theta}\left(\xi_{2 n+1}, \Im \xi_{2 n+1}\right)\right]}{1+\varpi_{\theta}\left(\wp \xi_{2 n}, \Im \xi_{2 n+1}\right)}, \\
\frac{\varpi_{\theta}\left(\xi_{2 n+1}, \wp \xi_{2 n}\right)\left[1+\varpi_{\theta}\left(\xi_{2 n}, \wp \xi_{2 n}\right)\right]}{1+\varpi_{\theta}\left(\xi_{2 n}, \xi_{2 n+1}\right)}
\end{array}\right\} \\
& =\max \left\{\begin{array}{c}
\varpi_{\theta}\left(\xi_{2 n}, \xi_{2 n+1}\right), \frac{\varpi_{\theta}\left(\xi_{2 n}, \xi_{2 n+1}\right)\left[1+\varpi_{\theta}\left(\xi_{2 n+1}, \xi_{2 n+2}\right)\right]}{1+\varpi_{\theta}\left(\xi_{2 n+1}, \xi_{2 n+2}\right)}, \\
\frac{\varpi_{\theta}\left(\xi_{2 n+1}, \xi_{2 n+1}\right)\left[1+\varpi_{\theta}\left(\xi_{2 n}, \xi_{2 n+1}\right)\right]}{1+\varpi_{\theta}\left(\xi_{2 n}, \xi_{2 n+1}\right)}
\end{array}\right\} \\
& =\max \left\{\varpi_{\theta}\left(\xi_{2 n}, \xi_{2 n+1}\right), \varpi_{\theta}\left(\xi_{2 n}, \xi_{2 n+1}\right), 0\right\} \\
& =\varpi_{\theta}\left(\xi_{2 n}, \xi_{2 n+1}\right) \text {, }
\end{aligned}
$$

and

$$
\begin{aligned}
& \Pi\left(\xi_{2 n}, \xi_{2 n+1}\right)=\min \left\{\varpi_{\theta}\left(\xi_{2 n}, \xi_{2 n+1}\right) \varpi_{\theta}\left(\xi_{2 n}, \wp \xi_{2 n}\right), \varpi_{\theta}\left(\xi_{2 n}, \wp \xi_{2 n}\right) \varpi_{\theta}\left(\xi_{2 n+1}, \Im \xi_{2 n+1}\right), \varpi_{\theta}\left(\xi_{2 n+1}, \wp \xi_{2 n}\right) \varpi_{\theta}\left(\xi_{2 n}, \Im \xi_{2 n+1}\right)\right\} \\
& \leq \min \left\{\oplus_{\theta}\left(\xi_{2 n}, \xi_{2 n+1}\right) \oplus_{\theta}\left(\xi_{2 n}, \xi_{2 n+1}\right), \oplus_{\theta}\left(\xi_{2 n}, \xi_{2 n+1}\right) \oplus_{\theta}\left(\xi_{2 n+1}, \xi_{2 n+2}\right), \oplus_{\theta}\left(\xi_{2 n+1}, \xi_{2 n+1}\right) \oplus_{\theta}\left(\xi_{2 n}, \xi_{2 n+2}\right)\right\}=0 \text {. }
\end{aligned}
$$

Applying (8) and (9) in (7), one can write $\Omega\left(\kappa \oplus_{\theta}\left(\xi_{2 n+1}, \xi_{2 n+2}\right)\right) \leq \Omega\left(\oplus_{\theta}\left(\xi_{2 n}, \xi_{2 n+1}\right)\right)$, By definition of $\Omega$, we conclude that have

Similarly, if we replace $\xi$ with $\xi_{2 n+2}$ and $\ell$ with $\xi_{2 n+3}$, we 
$\kappa \varpi_{\theta}\left(\xi_{2 n+2}, \xi_{2 n+3}\right) \leq \varpi_{\theta}\left(\xi_{2 n+1}, \xi_{2 n+2}\right), \quad$ for all $n \in \mathbb{N}$.

From (10) and (11), we get

$\varpi_{\theta}\left(\xi_{n}, \xi_{n+1}\right) \leq \nabla \varpi_{\theta}\left(\xi_{n-1}, \xi_{n}\right), \quad \nabla=\frac{1}{\kappa}<1$ for all $n \in \mathbb{N}$.
Now, by Lemma 1 , we observe that $\left\{\xi_{n}\right\}$ is Cauchy sequence. Since $\mathfrak{R}$ is complete, then there is $\varphi \in \mathfrak{R}$ such that $\limsup _{n \rightarrow \infty} \xi_{n}=\varphi$. Assume that $\varphi \notin \mathfrak{J}(\varphi)$, then we have

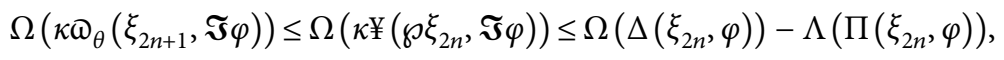

where

$$
\begin{aligned}
& \Delta\left(\xi_{2 n}, \varphi\right)=\max \left\{\varpi_{\theta}\left(\xi_{2 n}, \varphi\right), \frac{\varpi_{\theta}\left(\xi_{2 n}, \wp \xi_{2 n}\right)\left[1+\varpi_{\theta}(\varphi, \Im \varphi)\right]}{1+\varpi_{\theta}\left(\wp \xi_{2 n}, \Im \varphi\right)}, \frac{\varpi_{\theta}\left(\varphi, \wp \xi_{2 n}\right)\left[1+\varpi_{\theta}\left(\xi_{2 n}, \wp \xi_{2 n}\right)\right]}{1+\Phi_{\theta}\left(\xi_{2 n}, \varphi\right)}\right\}, \\
& \leq \max \left\{\varpi_{\theta}\left(\xi_{2 n}, \varphi\right), \frac{\Phi_{\theta}\left(\xi_{2 n}, \xi_{2 n+1}\right)\left[1+\varpi_{\theta}(\varphi, \Im \varphi)\right]}{1+\varpi_{\theta}\left(\xi_{2 n+1}, \Im \varphi\right)}, \frac{\varpi_{\theta}\left(\varphi, \xi_{2 n+1}\right)\left[1+\varpi_{\theta}\left(\xi_{2 n}, \xi_{2 n+1}\right)\right]}{1+\varpi_{\theta}\left(\xi_{2 n}, \varphi\right)}\right\} \\
& \leq \max \left\{\varpi_{\theta}\left(\xi_{2 n}, \varphi\right), \varpi_{\theta}\left(\xi_{2 n}, \xi_{2 n+1}\right)\left[1+\varpi_{\theta}(\varphi, \Im \varphi)\right], \varpi_{\theta}\left(\varphi, \xi_{2 n+1}\right)\left[1+\varpi_{\theta}\left(\xi_{2 n}, \xi_{2 n+1}\right)\right]\right\} .
\end{aligned}
$$

Taking limsup as $n \longrightarrow \infty$ in the above inequalities, we conclude that

$$
\limsup _{n \longrightarrow \infty} \Delta\left(\xi_{2 n}, \varphi\right)=0 \text { and } \limsup _{n \longrightarrow \infty} \Pi\left(\xi_{2 n}, \varphi\right)=0 .
$$

It follows from definition of $\Delta, \Pi$ and (11) that $\limsup _{n \longrightarrow \infty} \Omega\left(\kappa \varpi_{\theta}\left(\xi_{2 n+1}, \Im \varphi\right)\right)=0 \quad$ or $\quad \limsup _{n \longrightarrow \infty} \Phi_{\theta}$

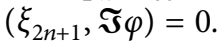

Using Lemma 2, we get

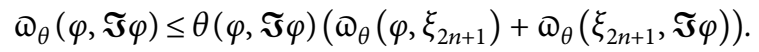

At the limit, we have $\varpi_{\theta}(\varphi, \Im \varphi) \longrightarrow 0$. Thus, $\varphi \in \Im \varphi$. Similarly, we can show that $\varphi \in \wp \varphi$. Hence, $\varphi$ is a cfp of the two mappings $\wp$ and $\mathfrak{J}$. For the uniqueness, let $\nu \neq \varphi$ be another cfp of $\wp$ and $\mathfrak{I}$, then, by our contractive condition, one can write

$$
\begin{aligned}
& \Omega\left(\kappa \varpi_{\theta}(\varphi, \nu)\right) \leq \Omega(\kappa ¥(\wp \varphi, \Im \nu)) \\
& \leq \Omega\left(\max \left\{\varpi_{\theta}(\varphi, \nu), \frac{\omega_{\theta}(\varphi, \wp \varphi)\left[1+\varpi_{\theta}(\nu, \Im \nu)\right]}{1+\varpi_{\theta}(\wp \varphi, \Im \nu)}, \frac{\omega_{\theta}(\nu, \wp \varphi)\left[1+\varpi_{\theta}(\varphi, \wp \varphi)\right]}{1+\Phi_{\theta}(\varphi, \nu)}\right\}\right)
\end{aligned}
$$

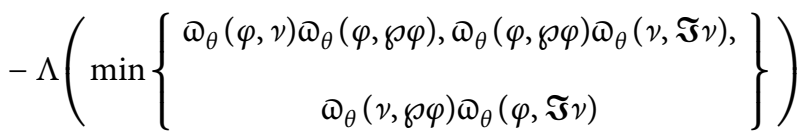

$$
\begin{aligned}
& \leq \Omega\left(\max \left\{\varpi_{\theta}(\varphi, \nu), \frac{\varpi_{\theta}(\varphi, \varphi)\left[1+\varpi_{\theta}(\nu, \nu)\right]}{1+\varpi_{\theta}(\varphi, \nu)}, \frac{\varpi_{\theta}(\nu, \varphi)\left[1+\varpi_{\theta}(\varphi, \varphi)\right]}{1+\varpi_{\theta}(\varphi, \nu)}\right\}\right) \\
& -\Lambda\left(\min \left\{\begin{array}{c}
\omega_{\theta}(\varphi, \nu) \varpi_{\theta}(\varphi, \varphi), \varpi_{\theta}(\varphi, \varphi) \varpi_{\theta}(\nu, \nu), \\
\varpi_{\theta}(\nu, \varphi) \varpi_{\theta}(\varphi, \nu)
\end{array}\right\}\right) \\
& =\Omega\left(\varpi_{\theta}(\varphi, \nu)\right)-\Lambda(0)=\Omega\left(\varpi_{\theta}(\varphi, \nu)\right) \text {. }
\end{aligned}
$$

This leads to $\Omega\left(\kappa \varpi_{\theta}(\varphi, \nu)\right) \leq \Omega\left(\varpi_{\theta}(\varphi, \nu)\right)$, or $(1-\kappa) \varpi_{\theta}(\varphi, \nu) \leq 0$; since $(1-\kappa) \nless 0$, then $\omega_{\theta}(\varphi, \nu)=0$. Thus, $\varphi=v$, i.e., the uniqueness holds. Then, the proof is completed. 
If we consider $\wp=\mathfrak{I}$ in the above theorem, we get the important below result.

Corollary 1. Let $\left(\mathfrak{R}, \Phi_{\theta}\right)$ be a complete extended $b$-metric space and $\wp: \mathfrak{R} \longrightarrow C B(\mathfrak{R})$ be a multivalued mapping such that, for all $\xi, \ell \in \mathfrak{R}$, the following hypothesis is fulfilled:
$\Omega(\kappa ¥(\wp \xi, \wp \ell)) \leq \Omega(\Delta(\xi, \ell))-\Lambda(\Pi(\xi, \ell))$,

where

$$
\begin{aligned}
& \Delta(\xi, \ell)=\max \left\{\omega_{\theta}(\xi, \ell), \frac{\omega_{\theta}(\xi, \wp \xi)\left[1+\Phi_{\theta}(\ell, \wp \ell)\right]}{1+\Phi_{\theta}(\wp \xi, \wp \ell)}, \frac{\omega_{\theta}(\ell, \wp \xi)\left[1+\omega_{\theta}(\xi, \wp \xi)\right]}{1+\omega_{\theta}(\xi, \ell)}\right\} \\
& \Pi(\xi, \ell)=\min \left\{\varpi_{\theta}(\xi, \ell) \varpi_{\theta}(\xi, \wp \xi), \varpi_{\theta}(\xi, \wp \xi) \varpi_{\theta}(\ell, \wp \ell), \varpi_{\theta}(\ell, \wp \xi) \varpi_{\theta}(\xi, \wp \ell)\right\}
\end{aligned}
$$

(i) $\Omega: \mathbb{R}^{+} \longrightarrow \mathbb{R}^{+}$is a nondecreasing and continuous function such that $\Omega(\tau)=0$ if $\tau=0$

(ii) $\Lambda: \mathbb{R}^{+} \longrightarrow \mathbb{R}^{+}$is a continuous function such that $\Lambda(\tau)=0$ if $\tau=0$

If $\lim _{n, m \rightarrow \infty} \Phi_{\theta}\left(\xi_{n}, \xi_{m}\right) \leq(1 / \kappa)$ with $\kappa>1$, then $\wp$ has a unique fixed point.

Example 3. Assume that $\mathfrak{R}=[0,1]$ and $1<e<\infty$. Define $\omega_{\theta}: \mathfrak{R} \times \mathfrak{R} \longrightarrow \mathbb{R}^{+}$by $\omega_{\theta}(\xi, \ell)=|\xi-\ell|^{e}$ for all $\xi, \ell \in \mathfrak{R}$, then the pair $\left(\Re, \Phi_{\theta}\right)$ is an extended $b$-metric space with the function $\theta(\xi, \ell)=2^{e-1}+((|\xi|+|\ell|) / 2), \quad$ see $\quad[20]$

Define $\wp, \mathfrak{I}: \mathfrak{R} \longrightarrow C B(\mathfrak{R})$ and $\Omega, \Lambda: \mathbb{R}^{+} \longrightarrow \mathbb{R}^{+}$by

$$
\begin{aligned}
& \wp \xi=\left[0, \frac{\sqrt[e]{\xi}}{\sqrt[e]{\xi \kappa(\mu+5)}}\right], \\
& \wp \ell=\left[0, \frac{\sqrt[e]{\ell}}{\sqrt[e]{\kappa(\mu+5)}}\right], \quad \text { for all } \mu \geq \kappa,
\end{aligned}
$$

and $\Omega(\omega)=\omega$ and $\Lambda(\omega)=((\mu+4) /(\mu+5)) \omega$, for any $\omega \in \mathfrak{R}$.

Now, we have

$$
\begin{aligned}
& \kappa ¥(\wp \xi, \Im \ell)=\kappa ¥\left(\left[0, \sqrt[e]{\frac{\xi}{\kappa(\mu+5)}}\right],\left[0, \sqrt[e]{\frac{\ell}{\kappa(\mu+5)}}\right]\right), \\
& \leq \kappa\left|\sqrt[e]{\frac{\xi}{\kappa(\mu+5)}}-\sqrt[e]{\frac{\xi}{\kappa(\mu+5)}}\right|^{e} \\
& =\kappa \frac{1}{\kappa(\mu+5)}|\xi-\ell|^{e} \\
& =\frac{1}{\mu+5}{\aleph_{\theta}}_{\theta}(\xi, \ell) \\
& \leq \frac{1}{\mu+5} \Delta(\xi, \ell) \\
& =\left(1-\frac{\mu+4}{\mu+5}\right) \Delta(\xi, \ell) \\
& =\Delta(\xi, \ell)-\left(\frac{\mu+4}{\mu+5}\right) \Delta(\xi, \ell) \\
& \leq \Omega(\Delta(\xi, \ell))-\Lambda(\Pi(\xi, \ell)) .
\end{aligned}
$$


Thus, all required conditions of Theorem 1 are fulfilled. Hence, $\wp$ and $\mathfrak{I}$ have a unique $\mathrm{cfp}$, which is 0 .

Theorem 2. Suppose that $\wp, \mathfrak{\Im}: \mathfrak{R} \longrightarrow C B(\mathfrak{R})$ are multivalued mappings defined on a complete extended $b$-metric space $\left(\mathfrak{R}, \oplus_{\theta}\right)$. Let for all $\xi, \ell \in \mathfrak{R}$,

$$
\kappa ¥(\wp \xi, \mathfrak{\jmath} \ell) \leq \sigma\left(\varpi_{\theta}(\xi, \ell)\right) \Delta(\xi, \ell)+\rho\left(\varpi_{\theta}(\xi, \ell)\right) \Pi(\xi, \ell),
$$

where $\Delta(\xi, \ell)$ and $\Pi(\xi, \ell)$ are defined in the above theorem and $\sigma(\xi), \rho(\xi) \in \Xi$ such that $\sigma(\xi)+\rho(\xi)<1$. If $\lim _{n, m \longrightarrow \infty} \Phi_{\theta}\left(\xi_{n}, \xi_{m}\right) \leq(1 / k), k>1$, then $\wp$ and have $a$ unique $c f p$.

Proof. For a fixed element $\xi \in \mathfrak{R}$, define $\xi^{\circ}=\xi$ and let $\xi_{1} \in \wp_{\xi^{\circ}}$, by Lemma 3 (for $\hbar=1$ ), there exists $\xi_{2} \in \Im \xi_{1}$ such that $\varpi_{\theta}\left(\xi_{1}, \xi_{2}\right) \leq ¥\left(\wp_{\xi^{\circ}}, \mathfrak{\Im} \xi_{1}\right)$. For $\xi_{2} \in \mathfrak{\Im} \xi_{1}$, there exists $\xi_{3} \in \wp \xi_{1}$ such that $\Phi_{\theta}\left(\xi_{2}, \xi_{3}\right) \leq ¥\left(\wp \xi_{1}, \Im \xi_{2}\right)$.

With the same scenario, we obtain that $\xi_{2 n+1} \in \wp \xi_{2 n}$, $\xi_{2 n+2} \in \mathfrak{\Im} \xi_{2 n+1}$. If for some $n, \xi_{2 n+1}=\xi_{2 n+2}$, then $\left\{\xi_{n}\right\}$ is a Cauchy sequence. Assume that, for each $n, \xi_{2 n+1} \neq \xi_{2 n+2}$. Then, by (22), we get

$$
\begin{aligned}
& \kappa \varpi_{\theta}\left(\xi_{2 n+1}, \xi_{2 n+2}\right) \leq \kappa ¥\left(\wp \xi_{2 n}, \Im \xi_{2 n+1}\right), \\
& \leq \sigma\left(\oplus_{\theta}\left(\xi_{2 n}, \xi_{2 n+1}\right)\right) \Delta\left(\xi_{2 n}, \xi_{2 n+1}\right)+\rho\left(\varpi_{\theta}\left(\xi_{2 n}, \xi_{2 n+1}\right)\right) \Pi\left(\xi_{2 n}, \xi_{2 n+1}\right),
\end{aligned}
$$

where $\Delta\left(\xi_{2 n}, \xi_{2 n+1}\right)=\Phi_{\theta}\left(\xi_{2 n}, \xi_{2 n+1}\right)$ and $\Pi\left(\xi_{2 n}, \xi_{2 n+1}\right)=0$ according to the above theorem.

It follows from (23) that

$$
\kappa ळ_{\theta}\left(\xi_{2 n+1}, \xi_{2 n+2}\right) \leq \sigma\left(\oplus_{\theta}\left(\xi_{2 n}, \xi_{2 n+1}\right)\right) \varpi_{\theta}\left(\xi_{2 n}, \xi_{2 n+1}\right) .
$$
write

Similarly, replacing $\xi$ with $\xi_{2 n+2}$ and $\ell$ with $\xi_{2 n+3}$, we can

$$
\kappa \varpi_{\theta}\left(\xi_{2 n+2}, \xi_{2 n+3}\right) \leq \sigma\left(\oplus_{\theta}\left(\xi_{2 n+1}, \xi_{2 n+2}\right)\right) \varpi_{\theta}\left(\xi_{2 n+1}, \xi_{2 n+2}\right) .
$$

From (24) and (25), we have

$$
\varpi_{\theta}\left(\xi_{n}, \xi_{n+1}\right) \leq \frac{1}{\kappa} \varlimsup_{\theta}\left(\xi_{n-1}, \xi_{n}\right) .
$$

From Lemma 1 , we obtain that $\left\{\xi_{n}\right\}$ is a Cauchy sequence. The completeness of $\mathfrak{R}$ leads to the conclusion that there is $\varphi \in \mathfrak{R}$ such that $\limsup _{n \longrightarrow \infty} \xi_{n}=\varphi$. Let $\varphi \notin \mathfrak{I}(\varphi)$, then, by Theorem 1 , one can write

$$
\begin{aligned}
& \omega_{\theta}\left(\xi_{2 n+1}, \Im \varphi\right) \leq \oplus_{\theta}\left(\wp \xi_{2 n}, \Im \varphi\right), \\
& \leq \sigma\left(\oplus_{\theta}\left(\xi_{2 n}, \varphi\right)\right) \Delta\left(\xi_{2 n}, \varphi\right)+\rho\left(\varpi_{\theta}\left(\xi_{2 n}, \varphi\right)\right) \Pi\left(\xi_{2 n}, \varphi\right) .
\end{aligned}
$$

Passing to the upper limit, we can get

$$
\limsup _{n \rightarrow \infty} \oplus_{\theta}\left(\xi_{2 n+1}, \mathfrak{\Im} \varphi\right) \leq 0 .
$$

By the fact $\omega_{\theta}\left(\xi_{2 n+1}, \Im \varphi\right) \geq 0$, one can see $\limsup _{n \longrightarrow \infty} \Phi_{\theta}\left(\xi_{2 n+1}, \Im \varphi\right) \geq 0$. Thus, we conclude that

$$
\limsup _{n \longrightarrow \infty} \Phi_{\theta}\left(\xi_{2 n+1}, \Im \varphi\right)=0 .
$$

From Lemma 2,

$$
\varpi_{\theta}(\varphi, \Im \varphi) \leq \theta(\varphi, \Im \varphi)\left(\varpi_{\theta}\left(\varphi, \xi_{2 n+1}\right)+\varpi_{\theta}\left(\xi_{2 n+1}, \Im \varphi\right)\right) .
$$

By taking the upper limit, we have $\varpi_{\theta}(\varphi, \Im \varphi) \longrightarrow 0$. Thus, $\varphi \in \mathfrak{J} \varphi$. Similarly, we can show that $\varphi \in \wp \varphi$. Hence, $\varphi$ is a cfp of $\wp$ and $\mathfrak{\Im}$. The uniqueness comes immediately in a similar way as in Theorem 1.

If we put $\wp=\mathfrak{I}$ in Theorem 2 , we have the following result.

Corollary 2. let $\wp: \mathfrak{R} \longrightarrow C B(\mathfrak{R})$ be a multivalued mapping defined on a complete extended $b$-metric space $\left(\Re, \varpi_{\theta}\right)$. Let for all $\xi, \ell \in \mathfrak{R}$,

$$
\kappa ¥(\wp \xi, \wp \ell) \leq \sigma\left(\varpi_{\theta}(\xi, \ell)\right) \Delta(\xi, \ell)+\rho\left(\varpi_{\theta}(\xi, \ell)\right) \Pi(\xi, \ell),
$$

where $\Delta(\xi, \ell)$ and $\Pi(\xi, \ell)$ are defined in Corollary 1 and $\sigma(\xi), \rho(\xi) \in \Xi \quad$ such that $\sigma(\xi)+\rho(\xi)<1$. If $\lim _{n, m \longrightarrow \infty} \omega_{\theta}\left(\xi_{n}, \xi_{m}\right) \leq(1 / \kappa), \kappa>1$, then $\wp$ has a unique fixed point.

Example 4. Suppose that $\mathfrak{R}=[0,1]$. Define $\omega_{\theta}: \mathfrak{R} \times \mathfrak{R} \longrightarrow \mathbb{R}^{+}$by $\varpi_{\theta}(\xi, \ell)=|\xi-\ell|^{2}$ for all $\xi, \ell \in \mathfrak{R}$, then the pair $\left(\Re, \Phi_{\theta}\right)$ is an extended $b$-metric space with the function $\theta: \mathfrak{R} \times \mathfrak{R} \longrightarrow[1, \infty)$, which takes the form $\theta(\xi, \ell)=2+((|\xi|+|\ell|) / 2)$. Define $\wp, \mathfrak{\Im}: \mathfrak{R} \longrightarrow C B(\mathfrak{R})$ and $\sigma, \rho: \mathbb{R}^{+} \longrightarrow[0,1)$ as follows:

$$
\begin{aligned}
& \wp \xi=\left[\frac{\xi}{5 \kappa}, \frac{\xi}{3 \kappa}\right], \\
& \Im \ell=\left[\frac{\ell}{5 \kappa}, \frac{\ell}{3 \kappa}\right], \quad \text { for all } \xi, \ell \in \Re, \kappa>1 .
\end{aligned}
$$

and $\sigma(\omega)=\rho(\omega)=(1 / 9)<1$, for all $\omega \in \mathfrak{R}$. Consider 


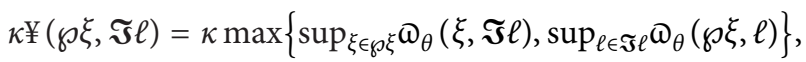

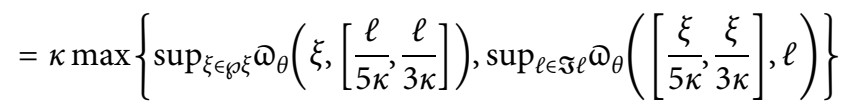

$$
\begin{aligned}
& =\kappa \max \left\{\left|\frac{\xi}{5 \kappa}-\frac{\ell}{5 \kappa}\right|^{2},\left|\frac{\xi}{3 \kappa}-\frac{\ell}{3 \kappa}\right|^{2}\right\} \\
& \leq \frac{\kappa}{9 \kappa^{2}} \max \left\{|\xi-\ell|^{2},|\xi-\ell|^{2}\right\} \\
& \leq \frac{1}{9} \max \left\{|\xi-\ell|^{2},|\xi-\ell|^{2}\right\},\left(\text { since } \frac{1}{\kappa}<1\right) \\
& \leq \frac{1}{9} \varpi_{\theta}(\xi, \ell) \\
& \leq \sigma\left(\varpi_{\theta}(\xi, \ell)\right) \Delta(\xi, \ell) \\
& \leq \sigma\left(\aleph_{\theta}(\xi, \ell)\right) \Delta(\xi, \ell)+\rho\left(\aleph_{\theta}(\xi, \ell)\right) \Pi(\xi, \ell) .
\end{aligned}
$$

Hence, the conditions managed by Theorem 2 are fulfilled, thereby concluding $0 \in \mathfrak{R}$ is the unique $\operatorname{cfp}$ of $\wp$ and $\mathfrak{\Im}$.

$$
\begin{aligned}
& \wp \xi=\left[0, \frac{\xi}{\sqrt{8 \kappa}}\right], \\
& \Im \ell=\left\{\frac{\ell}{\sqrt{8 \kappa}}\right\}, \quad \forall \xi, \ell \in \mathfrak{R}, \kappa>1,
\end{aligned}
$$

Example 5. Suppose that all data of Example 4 are fulfilled. Define the multivalued mappings $\wp, \mathfrak{\Im}: \mathfrak{R} \longrightarrow C B(\mathfrak{R})$ and $\sigma, \rho: \mathbb{R}^{+} \longrightarrow[0,1)$ by and $\sigma(\omega)=\rho(\omega)=(1 / 8)<1$, for all $\omega \in \mathfrak{R}$. Consider

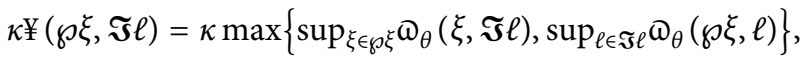

$$
\begin{aligned}
& =\kappa \max \left\{\sup _{\xi \in € \xi} \varpi_{\theta}\left(\xi, \frac{\ell}{\sqrt{8 \kappa}}\right), \sup _{\ell \in \Im \ell}{\varpi_{\theta}}_{\theta}\left(\left[0, \frac{\xi}{\sqrt{8 \kappa}}\right], \ell\right)\right\} \\
& =\kappa \max \left\{\left|\frac{\xi}{\sqrt{8 \kappa}}-\frac{\ell}{\sqrt{8 \kappa}}\right|^{2},\left|\frac{\ell}{\sqrt{8 \kappa}}\right|^{2}\right\} \\
& =\frac{\kappa}{8 \kappa} \max \left\{|\xi-\ell|^{2},|\ell|^{2}\right\} \\
& \leq \frac{1}{8} \max \left\{|\xi-\ell|^{2},\left|\ell-\frac{\ell}{\sqrt{8 \kappa}}\right|^{2}\right\} \\
& \leq \frac{1}{8} \max \left\{\varpi_{\theta}(\xi, \ell), \varpi_{\theta}(\ell, \Im \ell)\right\} \\
& \leq \sigma\left(\aleph_{\theta}(\xi, \ell)\right) \Delta(\xi, \ell) \\
& \leq \sigma\left(\aleph_{\theta}(\xi, \ell)\right) \Delta(\xi, \ell)+\rho\left(\aleph_{\theta}(\xi, \ell)\right) \Pi(\xi, \ell) .
\end{aligned}
$$


Hence, the conditions managed by Theorem 2 are fulfilled, thereby concluding $0 \in \mathfrak{R}$ is the unique cfp of $\wp$ and $\mathfrak{\Im}$.

\section{Solving a Fractional Nonlinear Differential Equation}

Recently, by the technique of nonlinear analysis such as fixed-point results, the Leray-Schauder theorem and stability, there are some papers dealing with the existence of solutions of nonlinear initial-value problems of fractional differential equations (see [21-23]). The main advantage of using fractional nonlinear differential equations is to describe the dynamics of complex nonlocal systems with memory. This part is devoted to obtain an existence solution of the subsequent nonlinear differential equation of fractional order:

$$
\complement^{\complement} \mathscr{D}^{\vartheta} \xi(\tau)=\Theta(\tau, \xi(\tau)), \quad \tau \in(0,1), \vartheta \in(1,2],
$$

with boundary conditions

$$
\begin{aligned}
\xi(0)=0, & \\
\xi(1)=\int_{0}^{\alpha} \xi(\mu) \mathrm{d} \mu, & \alpha \in(0,1) .
\end{aligned}
$$

The Caputo fractional derivative ${ }^{C} D{ }^{\vartheta}$ with ordered $\vartheta$ is defined as follows:

$$
{ }^{\complement} D^{\vartheta} \Theta(\tau)=\frac{1}{\Gamma(v-\vartheta)} \int_{0}^{\tau}(\tau-\mu)^{v-\vartheta-1} \Theta^{v}(\mu) \mathrm{d} \mu,
$$

where $v-1 \leq \vartheta<v, v=[\vartheta]+1$, and $\Theta:[0,1] \times \mathbb{R} \longrightarrow \mathbb{R}^{+}$is a continuous function. Let $\mathfrak{R}=C[0,1]$ be the set of all realvalued continuous functions on $[0,1]$. Define $\varpi_{\theta}: \mathfrak{R} \times \mathfrak{R} \longrightarrow \mathbb{R}$ and $\theta: \mathfrak{R} \times \mathfrak{R} \longrightarrow[1, \infty)$ by

$$
ळ_{\theta}(\xi, \ell)=\left(\sup _{\tau \in[0,1]}|\xi(\tau)-\ell(\tau)|\right)^{e} \text { and } \theta(\xi, \ell)=2^{\mathrm{e}-1}+\frac{|\xi(\tau)|+|\ell(\tau)|}{1+|\xi(\tau)|+|\ell(\tau)|}
$$

for all $\xi, \ell \in \mathfrak{R}, e>1$. Then, the pair $\left(\mathfrak{R}, \oplus_{\theta}\right)$ is a complete extended $b$-metric space [20]. Here, we need to be reminded that the Riemann-Liouville fractional integral of order $\vartheta$ is as follows:

$$
I^{\vartheta} \Theta(\tau)=\frac{1}{\Gamma(\vartheta)} \int_{0}^{\tau}(\tau-\mu)^{9-1} \Theta(\mu) \mathrm{d} \mu
$$

Theorem 3. The problem (36) with boundary conditions (37) has a unique solution if the following assumptions are fulfilled:

(i) $\Theta:[0,1] \times \mathbb{R} \longrightarrow \mathbb{R}^{+}$is a continuous function satisfying

Now, our main theorem of this section is.

$$
|\Theta(\tau, \xi)-\Theta(\tau, \ell)| \leq \sqrt{[e]} \phi|\xi-\ell|, \quad \text { for all } \tau \in[0,1], \xi, \ell \in \Re, e>1
$$

(ii) There is a constant $\phi$ such that $\phi G<1$, where

(iii) $\lim _{n, m \longrightarrow \infty} \Phi_{\theta}\left(\xi_{n}, \xi_{m}\right) \leq(1 / \kappa)$, where $\kappa=2^{e-1}$.

$$
\mathrm{G}=\left(\frac{\left(2-\alpha^{2}\right)(1+\vartheta)+2 \tau\left(\vartheta+1+\alpha^{\vartheta+1}\right)}{\left(2-\alpha^{2}\right) \Gamma(\vartheta+2)}\right)^{e}, \quad \alpha \in(0,1) \text {. }
$$

Proof. Define the mapping $\wp: \mathfrak{R} \longrightarrow \Re$ by

$$
\begin{aligned}
\wp \xi(\tau)= & \frac{2 \tau}{\left(2-\alpha^{2}\right) \Gamma(\vartheta)} \int_{0}^{\alpha} \int_{0}^{\mu}(\mu-\sigma)^{\vartheta-1} \Theta(\sigma, \xi(\sigma)) \mathrm{d} \sigma \mathrm{d} \mu-\frac{2 \tau}{\left(2-\alpha^{2}\right) \Gamma(\vartheta)} \int_{0}^{1}(1-\mu)^{\vartheta-1} \Theta(\mu, \xi(\mu)) \mathrm{d} \mu+\frac{1}{\Gamma(\vartheta)} \\
& \int_{0}^{\tau}(\tau-\mu)^{9-1} \Theta(\mu, \xi(\mu)) \mathrm{d} \mu,
\end{aligned}
$$

For $\tau \in[0,1]$. The function $\xi \in \mathfrak{R}$ is a unique solution of problem (36) if $\xi \in \wp \xi$, i.e., $\xi$ is a unique fixed point of the multivalued mapping $\wp$. To get that, we shall prove that $\wp$ satisfies the contractive condition of Corollary 1. Consider 


$$
\begin{aligned}
& 2^{e-1} \varpi_{\theta}(\wp \xi(\tau), \wp \ell(\tau))=2^{e-1}\left(\sup _{\tau \in[0,1]}|\wp \xi(\tau)-\wp \ell(\tau)|^{e}\right) \\
& =2^{e-1}\left(\sup _{\tau \in[0,1]} \mid \frac{2 \tau}{\left(2-\alpha^{2}\right) \Gamma(\vartheta)} \int_{0}^{\alpha} \int_{0}^{\mu}(\mu-\sigma)^{9-1} \Theta(\sigma, \xi(\sigma)) \mathrm{d} \sigma \mathrm{d} \mu-\frac{2 \tau}{\left(2-\alpha^{2}\right) \Gamma(\vartheta)} \int_{0}^{1}(1-\mu)^{9-1} \Theta(\mu, \xi(\mu)) \mathrm{d} \mu\right. \\
& +\frac{1}{\Gamma(\vartheta)} \int_{0}^{\tau}(\tau-\mu)^{9-1} \Theta(\mu, \xi(\mu)) \mathrm{d} \mu-\frac{2 \tau}{\left(2-\alpha^{2}\right) \Gamma(\vartheta)} \int_{0}^{\alpha} \int_{0}^{\mu}(\mu-\sigma)^{9-1} \Theta(\sigma, \ell(\sigma)) \mathrm{d} \sigma \mathrm{d} \mu \\
& \left.+\frac{2 \tau}{\left(2-\alpha^{2}\right) \Gamma(\vartheta)} \int_{0}^{1}(1-\mu)^{9-1} \Theta(\mu, \ell(\mu)) \mathrm{d} \mu-\left.\frac{1}{\Gamma(\vartheta)} \int_{0}^{\tau}(\tau-\mu)^{9-1} \Theta(\mu, \ell(\mu)) \mathrm{d} \mu\right|^{e}\right) \\
& \leq 2^{e-1}\left(\operatorname { s u p } _ { \tau \in [ 0 , 1 ] } \left(\frac{2 \tau}{\left(2-\alpha^{2}\right) \Gamma(\vartheta)} \int_{0}^{\alpha} \int_{0}^{\mu}(\mu-\sigma)^{9-1}|\Theta(\sigma, \xi(\sigma))-\Theta(\sigma, \ell(\sigma))| \mathrm{d} \sigma d \mu\right.\right. \\
& +\frac{2 \tau}{\left(2-\alpha^{2}\right) \Gamma(9)} \int_{0}^{1}(1-\mu)^{9-1}|\Theta(\mu, \ell(\mu))-\Theta(\mu, \xi(\mu))| \mathrm{d} \mu \\
& \left.\left.+\frac{1}{\Gamma(\vartheta)} \int_{0}^{\tau}(\tau-\mu)^{9-1}|\Theta(\mu, \xi(\mu))-\Theta(\mu, \ell(\mu))| \mathrm{d} \mu\right)^{e}\right) \\
& \leq 2^{e-1}\left(\operatorname { s u p } _ { \tau \in [ 0 , 1 ] } \left(\frac{2 \tau}{\left(2-\alpha^{2}\right) \Gamma(\vartheta)} \int_{0}^{\alpha} \int_{0}^{\mu}(\mu-\sigma)^{9-1} \mathrm{~d} \sigma \mathrm{d} \mu\right.\right. \\
& \left.\left.+\frac{2 \tau}{\left(2-\alpha^{2}\right) \Gamma(\vartheta)} \int_{0}^{1}(1-\mu)^{9-1} \mathrm{~d} \mu+\frac{1}{\Gamma(\vartheta)} \int_{0}^{\tau}(\tau-\mu)^{9-1} \mathrm{~d} \mu\right)^{e} \phi|\xi-\ell|^{e}\right) \\
& \leq 2^{e-1} \phi\left\{\frac{\left(2-\alpha^{2}\right)(1+\vartheta)+2 \tau\left(\vartheta+1+\alpha^{9+1}\right)}{\left(2-\alpha^{2}\right) \Gamma(\vartheta+2)}\right\}^{e} \times\left(\sup _{\tau \in[0,1]}|\xi(\tau)-\ell(\tau)|\right)^{e} \\
& =2^{e-1} \phi \mathrm{G} \varpi_{\theta}(\xi(\tau), \ell(\tau)) \\
& \leq 2^{e-1} \varpi_{\theta}(\xi(\tau), \ell(\tau)) \text {. }
\end{aligned}
$$

This implies that

$$
\begin{aligned}
2^{e-1} \varpi_{\theta}(\wp \xi(\tau), \wp \ell(\tau)) & \leq 2^{e-1} \varpi_{\theta}(\xi(\tau), \ell(\tau)), \\
& \leq 2^{e-1} \Delta(\xi, \ell) \\
& \leq \Omega(\Delta(\xi, \ell))-\Lambda(\Pi(\xi, \ell)),
\end{aligned}
$$

where $\kappa=2^{e-1}, \Omega(\varkappa)=4^{e-1} \varkappa$, and $\Lambda(\varkappa)=\left(\varkappa / 16^{e-1}\right)$. Then, by Corollary 1 , there exists a unique fixed point of the mapping (43), which is the unique solution of problem (36) in $\mathfrak{R}$.

\section{Solving a System of Nonlinear Fractional Differential Equations}

Differential equations of fractional order have been the focus of many studies due to their frequent appearance in various applications in fluid mechanics, viscoelasticity, physics, engineering, and biology. Recently, a large amount of literature studies developed concerning the application of fractional differential equations in nonlinear dynamics [24-29]. In this part, we shall find the existence of a solution to the following system of nonlinear fractional ordered differential equations:

$$
\left\{{ }^{\complement} D^{\vartheta} \beth(\tau)+\wp(\beth(\tau))=0, \tau \in[0,1], \vartheta \in(1,2],{ }^{\complement} D^{\vartheta} \beth(\tau)+\Im(\beth(\tau))=0, \tau \in[0,1], \vartheta \in(1,2], \beth(0)=\beth(0)=\Omega, \beth(1)=\beth(1)=\Omega^{*},\right.
$$

where $\Omega$ and $\Omega^{*}$ are constants, $\wp, \mathfrak{I}:[0,1] \times \mathbb{R}^{+} \longrightarrow \mathbb{R}^{+}$, and ${ }^{C} D^{9}$ refers to the Caputo fractional derivative. If we apply
Green's (continuous) function $\Upsilon(\tau, \mu)$ on $[0,1] \times[0,1],(18)$ is equivalent to the following system: 


$$
\begin{cases}\beth(\tau)=\aleph(\tau)+\int_{0}^{1} \Upsilon(\tau, \mu) \wp(\beth(\mu)) \mathrm{d} \mu, & \tau \in[0,1], \\ \beth(\tau)=\aleph(\tau)+\int_{0}^{1} \Upsilon(\tau, \mu) \Im(\beth(\mu)) \mathrm{d} \mu, & \tau \in[0,1],\end{cases}
$$

where $\Upsilon(\tau, \mu)$ is defined by

$$
\Upsilon(\tau, \mu)= \begin{cases}\frac{(\tau, \mu)^{\vartheta-1}-\tau(1-\mu)^{\vartheta-1}}{\Gamma(\vartheta)}, & 0 \leq \mu \leq \tau \leq 1, \\ \frac{-\tau(1-\mu)^{\vartheta-1}}{\Gamma(\vartheta)}, & 0 \leq \tau \leq \mu \leq 1 .\end{cases}
$$

Moreover, $\quad \sup _{\tau \in[0,1]} \int_{0}^{1}|\Upsilon(\tau, \mu)| \mathrm{d} \mu \leq 1$. Now, put $\phi_{1}(\tau, \mu, \xi(\mu))=\Upsilon(\tau, \mu) \wp(\beth(\mu))$ and $\phi_{2}(\tau, \mu, \ell(\mu))=$ $\Upsilon(\tau, \mu) \mathfrak{\Im}(\beth(\mu))$, then system (19) turns into

$$
\begin{cases}\xi(\tau)=\aleph(\tau)+\int_{0}^{1} \phi_{1}(\tau, \mu, \xi(\mu)) \mathrm{d} \mu, & \tau \in[0,1], \\ \ell(\tau)=\aleph(\tau)+\int_{0}^{1} \phi_{2}(\tau, \mu, \ell(\mu)) \mathrm{d} \mu, & \tau \in[0,1] .\end{cases}
$$

Let $\mathfrak{R}=C[0,1]$ be the set of all real-valued continuous functions on $[0,1]$. Define $\varpi_{\theta}: \mathfrak{R} \times \mathfrak{R} \longrightarrow \mathbb{R}$ and $\theta: \Re \times$ $\mathfrak{R} \longrightarrow[1, \infty)$ by

$$
\omega_{\theta}(\xi, \ell)=\left(\sup _{\tau \in[0,1]}|\xi(\tau)-\ell(\tau)|\right)^{e} \text { and } \theta(\xi, \ell)=2^{\mathrm{e}-1}+\frac{|\xi(\tau)|+|\ell(\tau)|}{1+|\xi(\tau)|+|\ell(\tau)|}
$$

for all $\xi, \ell \in \mathfrak{R}, e>1$. Then, the pair $\left(\mathfrak{R}, \Phi_{\theta}\right)$ is a complete extended $b$-metric space.

Now, we provide the following theorem to derive an existence result for the solution of problem (49).

Theorem 4. Consider system (49) under the following hypotheses: (i) $\phi_{1}, \phi_{2}:[0,1] \times[0,1] \times \mathbb{R}^{+} \longrightarrow \mathbb{R}^{+}$and $\aleph:[0,1] \longrightarrow \mathbb{R}^{+}$is continuous.

(ii) There is a continuous function $\Psi:[0,1] \times[0,1] \longrightarrow$ $\mathbb{R}^{+}$such that

$$
\left|\phi_{1}(\tau, \mu, p)-\phi_{2}(\tau, \mu, p)\right| \leq \Psi(\tau, \mu)|p-q|, \quad \text { for all } \tau, \mu \in[0,1], p, q \in \mathfrak{R} \text {. }
$$

(iii) $\sup _{\tau \in[0,1]} \int_{0}^{1}|\Psi(\tau, \mu)|^{e} d \mu \leq 1$.

(iv) $\lim _{n, m \longrightarrow \infty} \Phi_{\theta}\left(\xi_{n}, \xi_{m}\right) \leq(1 / \kappa)$, where $\kappa=2^{e-1}$.

Then, problem (49) has a unique solution on $\mathfrak{R}$, which is considered as the unique solution to system (46).
Proof. Consider two multivalued mappings $\wp, \mathfrak{\Im}: \mathfrak{R} \longrightarrow \mathfrak{R}$ having the form

$$
\begin{aligned}
& \wp \xi(\tau)=\aleph(\tau)+\int_{0}^{1} \phi_{1}(\tau, \mu, \xi(\mu)) \mathrm{d} \mu, \quad \tau \in[0,1], \\
& \Im \ell(\tau)=\aleph(\tau)+\int_{0}^{1} \phi_{2}(\tau, \mu, \ell(\mu)) \mathrm{d} \mu, \quad \tau \in[0,1] .
\end{aligned}
$$


Hence, the unique cfp of the mappings $\wp$ and $\mathfrak{I}$ defined in (52) is considered as the unique solution of problem (49), which leads to the solution of problem (47) and from it to the solution of system (46).

Consider

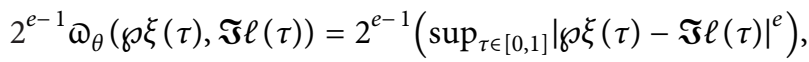

$$
\begin{aligned}
& =2^{e-1}\left(\sup _{\tau \in[0,1]}\left|\aleph(\tau)+\int_{0}^{1} \phi_{1}(\tau, \mu, \xi(\mu)) \mathrm{d} \mu-\aleph(\tau)-\int_{0}^{1} \phi_{2}(\tau, \mu, \ell(\mu)) \mathrm{d} \mu\right|^{e}\right) \\
& \leq 2^{e-1}\left(\sup _{\tau \in[0,1]} \int_{0}^{1}\left|\phi_{1}(\tau, \mu, \xi(\mu))-\phi_{2}(\tau, \mu, \ell(\mu))\right| \mathrm{d} \mu\right)^{e} \\
& \leq 2^{e-1}\left(\sup _{\tau \in[0,1]} \int_{0}^{1} \Psi(\tau, \mu)|\xi(\mu)-\ell(\mu)| \mathrm{d} \mu\right)^{e} \\
& \leq 2^{e-1}\left(\sup _{\tau \in[0,1]} \int_{0}^{1} \Psi(\tau, \mu) \mathrm{d} \mu\right)^{e}\left(\sup _{\tau \in[0,1]}|\xi(\tau)-\ell(\tau)|\right)^{e} \\
& \leq 2^{e-1} \varpi_{\theta}(\xi(\tau), \ell(\tau)) \\
& \leq 2^{e-1} \Delta(\xi, \ell) \\
& \leq \Omega(\Delta(\xi, \ell))-\Lambda(\Pi(\xi, \ell))),
\end{aligned}
$$

where $\kappa=2^{e-1}, \Omega(\varkappa)=4^{e-1} \mathcal{x}$, and $\Lambda(\varkappa)=\left(\varkappa / 16^{e-1}\right)$. Thus, by Theorem 1, there exists a unique cfp of the mappings (52), which is the unique solution of system (46) in $\mathfrak{R}$.

\section{Solving a Two-Dimensional Linear Fredholm Integral Equation}

Two-dimensional Fredholm integral equations of the second kind arise from many problems in engineering and mechanics by a suitable transformation. For example, in the calculation of plasma physics, it is usually required to solve Fredholm integral equations (see [30]).

In this section, we consider the two-dimensional Fredholm integral equation of the form:

$$
\xi(\tau, \mu)=¥(\tau, \mu)+\int_{0}^{1} \int_{0}^{1} \mathrm{G}(\tau, \mu, h, g) \Phi(\tau, \mu, \xi(h, g)) \mathrm{d} h \mathrm{~d} g ; \quad(\tau, \mu) \in[0,1]^{2},
$$

where $¥$ and $\mathrm{G}$ and $\Phi$ are given continuous functions defined on $L^{2}(C([0,1] \times[0,1]))$ and $\xi(h, g)$ is unknown on $L^{2}(C([0,1] \times[0,1]))$.
Assume that $\mathfrak{R}=C[0,1]$ is the set of all real-valued continuous functions on $[0,1]$. Define $\varpi_{\theta}: \Re \times \Re \longrightarrow \mathbb{R}$ and $\theta: \Re \times \Re \longrightarrow[1, \infty)$ by

$$
\varpi_{\theta}(\xi, \ell)=\left(\max _{\tau \in[0,1]}|\xi(\tau)-\ell(\tau)|\right)^{2} \text { and } \theta(\xi, \ell)=2+\frac{|\xi(\tau)|+|\ell(\tau)|}{1+|\xi(\tau)|+|\ell(\tau)|}
$$

for all $\xi, \ell \in \Re$. Then, the pair $\left(\Re, \varpi_{\theta}\right)$ is a complete extended $b$-metric space.

Now, we consider problem (46) under the following assumptions: (i) G: $[0,1]^{4} \longrightarrow \mathbb{R}^{+}$, and $\Phi:[0,1]^{2} \times \mathbb{R}^{+} \longrightarrow \mathbb{R}^{+}$and $¥:[0,1]^{2} \longrightarrow \mathbb{R}^{+}$are continuous functions.

(ii) For all $\xi, \ell \in \mathfrak{R}$,

$$
|\Phi(\tau, \mu, \xi(h, g))-\Phi(\tau, \mu, \ell(h, g))| \leq \frac{\sigma^{(1 / 2)}\left(\bigotimes_{\theta}(\xi(h, g), \ell(h, g))\right)}{\sqrt{2}}(|\xi(h, g)-\ell(h, g)|)
$$


(iii) There is a constant $z \in(0,1)$ such that $\int_{0}^{1} \int_{0}^{1} \mathrm{G}(\tau, \mu, h, g) \mathrm{d} h \mathrm{~d} g \leq z$.

(iv) $\lim _{n, m \longrightarrow \infty} \oplus_{\theta}\left(\xi_{n}, \xi_{m}\right) \leq(1 / 2)$.

Our main theorem in this section is presented as follows.

Theorem 5. Problem (54) has a unique solution on $L^{2}(C([0,1] \times[0,1]))$ if the hypotheses $\left(i_{1}-i v_{4}\right)$ hold.

Proof. As usual, define the multivalued operator $\wp: \mathfrak{R} \longrightarrow \mathfrak{R}$ by

$$
\wp(\xi(\tau, \mu))=¥(\tau, \mu)+\int_{0}^{1} \int_{0}^{1} \mathrm{G}(\tau, \mu, h, g) \Phi(\tau, \mu, \xi(h, g)) \mathrm{d} h \mathrm{~d} g ; \quad(\tau, \mu) \in[0,1] \times[0,1] .
$$

Then, we have

$$
\begin{aligned}
2|\wp(\xi(\tau, \mu))-\wp(\ell(\tau, \mu))|^{2} & =2\left(\int_{0}^{1} \int_{0}^{1} \mathrm{G}(\tau, \mu, h, g) \Phi(\tau, \mu, \xi(h, g)) \mathrm{d} h \mathrm{~d} g\right)-\left.\left(\int_{0}^{1} \int_{0}^{1} \mathrm{G}(\tau, \mu, h, g) \Phi(\tau, \mu, \ell(h, g)) \mathrm{d} h \mathrm{~d} g\right)\right|^{2}, \\
& \leq 2\left(\int_{0}^{1} \int_{0}^{1} \mathrm{G}(\tau, \mu, h, g)|\Phi(\tau, \mu, \xi(h, g))-\Phi(\tau, \mu, \ell(h, g))| \mathrm{d} h \mathrm{~d} g\right)^{2} \\
& \leq 2\left(\int_{0}^{1} \int_{0}^{1} \mathrm{G}(\tau, \mu, h, g) \mathrm{d} h \mathrm{~d} g\right)^{2}(|\Phi(\tau, \mu, \xi(h, g))-\Phi(\tau, \mu, \ell(h, g))|)^{2} \\
& \leq 2 z^{2}\left(\frac{\sigma^{(1 / 2)}\left(\oplus_{\theta}(\xi(h, g), \ell(h, g))\right)}{\sqrt{2}}(|\xi(h, g)-\ell(h, g)|)\right)^{2} \\
& \leq \sigma\left(\varpi_{\theta}(\xi(h, g), \ell(h, g))\right)|\xi(h, g)-\ell(h, g)|^{2} .
\end{aligned}
$$

Taking the maximum, we have

$$
\begin{aligned}
2\left(\max |\wp(\xi(\tau, \mu))-\wp(\ell(\tau, \mu))|^{2}\right) & \leq \sigma\left(\varpi_{\theta}(\xi(h, g), \ell(h, g))\right) \max \left\{|\xi(h, g)-\ell(h, g)|^{2}\right\}, \\
& =\sigma\left(\varpi_{\theta}(\xi(h, g), \ell(h, g))\right) \varpi_{\theta}(\xi(h, g), \ell(h, g)) \\
& \leq \sigma\left(\varpi_{\theta}(\xi, \ell)\right) \Delta(\xi, \ell)+\rho\left(\varpi_{\theta}(\xi, \ell)\right) \Pi(\xi, \ell) .
\end{aligned}
$$

By taking $\kappa=2$, from Corollary 2, operator (57) has a unique fixed point in $L^{2}(C([0,1] \times[0,1]))$, which is considered the unique solution of problem (54).

\section{Conclusion}

In this manuscript, we gave some common fixed point theorems involving generalized multivalued contraction mappings in the class of extended $b$-metric spaces. Applying our obtained results, we ensure the existence of (unique) solutions of a fractional differential equation, a system of fractional differential equations and a two-dimensional linear Fredholm integral equation. This affirms the utility of fixed point theory in the framework of fractional calculus.

\section{Data Availability}

The data used to support the findings of this study are available from the corresponding author upon request.

\section{Conflicts of Interest}

The authors declare that they have no conflicts of interest.

\section{Authors' Contributions}

All authors contributed equally and significantly in writing this article. 


\section{Acknowledgments}

The authors are grateful to the Spanish Government and the European Commission under grant no. IT1207-19. This work was supported in part by the Basque Government under grant no. IT1207-19.

\section{References}

[1] A. A. Kilbas, H. M. Srivastava, and J. J. Trujillo, "Theory and applications of fractional differential equations," North-Holland Mathematics Studies, Vol. 204, Elsevier Science B.V., Amsterdam, The Netherland, 2006.

[2] S. G. Samko, A. A. Kilbas, and O. I. Marichev, Fractional Integrals and Derivatives, Gordon and Breach, Yverdon, Switzerland, 1993.

[3] G. Wang, S. Liu, R. P. Agarwal, and L. Zhang, "Positive solutions of integral boundary value problem involving riemann-liouville fractional derivative," Journal of Fractional Calculus and Applications, vol. 4, no. 2, pp. 312-321, 2013.

[4] Y. Watanabe, "Notes on the generalized derivative of riemann-liouville and its application to leibnitz's formula, II," Tohoku Mathematical Journal, vol. 34, pp. 28-41, 1999.

[5] H. Aydi, M. Jleli, and B. Samet, "On positive solutions for a fractional thermostat model with a convex-concave source term via- $\psi$ Caputo fractional derivative," Mediterranean Journal of Mathematics, vol. 17, no. 1, pp. 1-6, 2020.

[6] H. R. Marasi and H. Aydi, "Existence and uniqueness results for two-term nonlinear fractional differential equations via a fixed point technique," Journal of Mathematics, vol. 2021, Article ID 6670176, 7 pages, 2021.

[7] S. G. Samko, A. A. Kilbas, and O. I. Marichev, Fractional Integrals and Derivatives: Theory and Applications, Gordon and Breach, Amsterdam, The Netherland, 1993.

[8] J. L. Lavoie, T. J. Osler, and R. Tremblay, "Frational derivatives and special functions," SIAM Review, vol. 18, no. 2, pp. $240-268,1976$.

[9] A. Fernandez, A Complex Analysis Approach to AtanganaBaleanu Fractional Calculus, Wiley, Hoboken, NJ, USA, 2019.

[10] F. Martínez, I. Martínez, M. K. A. Kaabar, R. Ortíz-Munuera, and S. Paredes, "Note on the conformable fractional derivatives and integrals of complex-valued functions of a real variable," IAENG International Journal of Applied Mathematics, vol. 50, no. 3, pp. 1-7, 2020.

[11] T. Kamran, M. Samreen, and Q. UL Ain, "A generalization of $b$-metric space and some fixed point theorems," Mathematics, vol. 5, no. 19, pp. 1-7, 2017.

[12] B. Alqahtani, A. Fulga, and E. Karapınar, "Non-unique fixed point results in extended B-metric space $b$ - metric space," Mathematics, vol. 6, no. 5, p. 68, 2018.

[13] N. Mlaiki, N. Souayah, T. Abdeljawad, and H. Aydi, "A new extension to the controlled metric type spaces endowed with a graph," Advances in Difference Equations, vol. 94, 2021.

[14] P. S. Kumari, E. Karapinar, and A. Atangana, "A numerical schemes and comparisons for fixed point results with applications to the solutions of Volterra integral equations in dislocated extended b-metric space," Alexandria Engineering Journal, vol. 59, no. 2, pp. 815-827, 2020.

[15] N. Alamgir, Q. Kiran, H. Isik, and H. Aydi, "Fixed point results via a Hausdorff controlled type metric," Advances in Difference Equations, vol. 24, 2020.

[16] T. Abdeljawad, R. P. Agarwal, E. Karapınar, and P. S. Kumari, "Solutions of the nonlinear integral equation and fractional differential equation using the technique of a fixed point with a numerical experiment in extended b-metric space," Symmetry, vol. 11, no. 5, p. 686, 2019.

[17] M. A. Geraghty, "On contractive mappings," Proceedings of the American Mathematical Society, vol. 40, no. 2, p. 604, 1973.

[18] S. Czerwik, "Nonlinear set-valued contraction mappings in $b$ metric spaces," Atti del Seminario Matematico e Fisico dell'Universita, vol. 46, no. 2, pp. 263-276, 1998.

[19] P. P. Murthy and H. K. Pathak, "Some fixed point theorems without continuity," Bulletin of the Calcutta Mathematical Society, vol. 82, pp. 212-215, 1990.

[20] M. S. Khan, Y. M. Singh, M. Abbas, and V. Rakočević, On nonunique fixed point of Ćiric type operators in extended b-metric spaces and applications, Rendiconti del Circolo Matematico di Palermo Series 2, Springer, Berlin, Germany, 2020.

[21] S. Zhang, "The existence of a positive solution for a nonlinear fractional differential equation," Journal of Mathematical Analysis and Applications, vol. 252, no. 2, pp. 804-812, 2000.

[22] Q. M. Al-Mdallal, M. I. Syam, and M. N. Anwar, "A collocation-shooting method for solving fractional boundary value problems," Communications in Nonlinear Science and Numerical Simulation, vol. 15, no. 12, pp. 3814-3822, 2010.

[23] C. P. Li and F. R. Zhang, "A survey on the stability of fractional differential equations," The European Physical Journal Special Topics, vol. 193, no. 1, pp. 27-47, 2011.

[24] X. Gao and J. Yu, "Synchronization of two coupled fractionalorder chaotic oscillators," Chaos, Solitons \& Fractals, vol. 26, no. 1, pp. 141-145, 2005.

[25] J.-H. He, "Approximate analytical solution for seepage flow with fractional derivatives in porous media," Computer Methods in Applied Mechanics and Engineering, vol. 167, no. 1-2, pp. 57-68, 1998.

[26] H. A. Hammad, H. Aydi, and N. Mlaiki, "Contributions of the fixed point technique to solve the $2 \mathrm{D}$ Volterra integral equations, Riemann-Liouville fractional integrals, and Atangana-Baleanu integral operators," Advances in Difference Equations, vol. 97, 2021.

[27] I. Podlubny, Fractional Differential Equations, Academic Press, Cambridge, MA, USA, 1999.

[28] M. Mehmood, H. Aydi, M. U. Ali, F. Uddin, A. Shoaib, and M. De La Sen, "Solutions of integral equations via fixed-point results on orthogonal gauge structure," Mathematical Problems in Engineering, vol. 2021, Article ID 8387262, 11 pages, 2021.

[29] P. Agarwal, "Some inequalities involving Hadamard-type k -fractional integral operators $k$-fractional integral operators," Mathematical Methods in the Applied Sciences, vol. 40, no. 11, pp. 3882-3891, 2017.

[30] R. Farengo, Y. C. Lee, and P. N. Guzdar, "An electromagnetic integral equation: application to microtearing modes," Physics of Fluids, vol. 26, no. 12, pp. 3515-3523, 1983. 